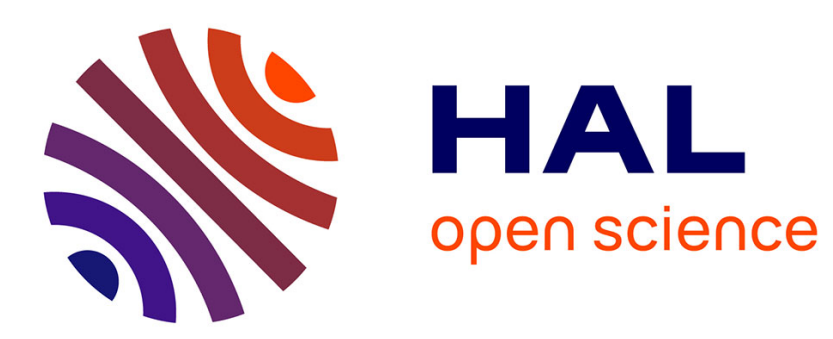

\title{
Du risque filicide au care: la transmission de la violence et son interruption dans l'œuvre de Doris Lessing
}

Françoise Hatchuel

\section{To cite this version:}

Françoise Hatchuel. Du risque filicide au care: la transmission de la violence et son interruption dans l'œuvre de Doris Lessing. Nouvelle revue de psychosociologie, 2017, 24. hal-01572225

\section{HAL Id: hal-01572225 \\ https://hal.science/hal-01572225}

Submitted on 10 Feb 2021

HAL is a multi-disciplinary open access archive for the deposit and dissemination of scientific research documents, whether they are published or not. The documents may come from teaching and research institutions in France or abroad, or from public or private research centers.
L'archive ouverte pluridisciplinaire HAL, est destinée au dépôt et à la diffusion de documents scientifiques de niveau recherche, publiés ou non, émanant des établissements d'enseignement et de recherche français ou étrangers, des laboratoires publics ou privés. 


\title{
Du risque filicide au care : la transmission de la violence et son interruption dans l'œuvre de Doris Lessing
}

\author{
Françoise Hatchuel
}

Comment le sujet peut-il s'éprouver ? Comment rendre compte de ce qui nous assaille, de ce à quoi nous faisons face et surtout, à l'heure de la rationalité triomphante, comment reconnaître la "part obscure " de nous-mêmes, le doute, I'ambivalence, l'incertitude, la complexité ? Comment exister comme sujets et non comme rouages remplaçables de procédures désubjectivantes (Diet, 2003) ? J'ai montré précédemment comment je considère que nos sociétés hypermodernes favorisent ce que j'ai appelé "I'illusion de l'éradication du doute " (Hatchuel, 2013a), à l'origine de bien des anxiétés contemporaines puisque le doute et l'ambivalence deviennent non plus une réalité à laquelle faire face mais des scories à éliminer.

Face à ce triomphe de l'emprise gestionnaire, la littérature, par sa puissance d'évocation et l'acuité de ses descriptions, peut nous être d'un précieux secours pour nommer l'existant, le reconnaître et l'assumer, c'est-à-dire in fine le faire vivre. "Les grands écrivains sont nos maîtres ", disait Freud, et ce notamment parce qu'ils nous font saisir de l'intérieur, en nous les faisant éprouver, la subtilité des processus psychiques. C'est en tout cas ce que je ressens à la lecture de Doris Lessing, prix Nobel 2007, dont l'écriture, en posant la multiplicité et la complexité de nos éprouvés comme un fait et non comme un problème, m'apparaît à la fois comme puissamment étayante (au sens de René Kaës, 1984), dans la mesure où elle permet au lecteur/à la lectrice de

Françoise Hatchuel, maîtresse de conférences habilitée à diriger les recherches, université Paris Nanterre, CREF (EA 1589) "Savoir, rapport au savoir et processus de transmission ". fhatchuel@orange.fr 
se reconnecter à lui/elle-même, et singulièrement éclairante sur la façon dont certains processus psychiques s'installent et se transmettent. Elle excelle notamment à mettre au jour la violence transmise dans laquelle elle se reconnaît en tant qu'enfant de deux parents eux-mêmes confrontés à une violence massive (elle est née en 1919 et ses parents ont été fortement marqués par la guerre). Je voudrais en particulier montrer comment son œuvre me permet de penser que cette transmission de la violence passe notamment par ce que j'appelle "I'angoisse du risque filicide ", c'est-à-dire la peur, pour le parent, de ne pas parvenir à soutenir l'investissement que demande le fait de faire grandir un enfant, et l'impossibilité d'assumer ses éprouvés de découragement. C'est en effet en animant des groupes de parole d'apprenant(e)s (Hatchuel, 2004) que j'ai repéré la peine, voire l'impossibilité, à exprimer et accueillir les difficultés d'apprentissage et que je l'ai interprétée comme un déni $d^{\prime}$ ordre anthropologique. Je pose l'hypothèse que ce déni vient soutenir le refus collectif d'imaginer que la transmission puisse échouer, dans la mesure où elle constitue un puissant rempart contre l'angoisse de mort. $C^{\prime}$ est pourquoi je donnerai également quelques éléments chronologiques sur la construction de l'œuvre, afin d'insister sur le temps et le travail nécessaires à la mise au jour de tels éprouvés.

\section{L'INLASSABLE TÂCHE DE VIVRE, ET DE VIVRE COMME FEMME}

La force de Doris Lessing est de rendre compte de I'inlassable travail psychique que demande le fait de vivre, et encore plus de vivre comme femme. Les femmes, en effet, sont en charge des soins aux enfants et, plus largement, du travail du care. Doris Lessing montre la façon dont celui-ci leur est imposé tout en étant minimisé, voire dénié, et la difficulté qui s'ensuit à en assumer la crainte de ne pas y arriver et l'épuisement qu'elle engendre. Je propose alors de parler de "risque filicide " en empruntant le terme " filicide " à Georges Gaillard (2001) mais en substituant " risque " à " haine " qui me semble trop sujette à malentendu. La notion de risque de désinvestissement, entendue au sens de Piera Aulagnier (1982; Hatchuel, 2012), me paraît en effet plus facile à appréhender de façon non culpabilisante. Or, c'est justement à partir de la culpabilité des mères que le processus de désinvestissement se répète. $C^{\prime}$ est donc le nœud pour l'interrompre.

Il faut en effet entendre que le désinvestissement (et le rejet qu'il implique) n'est pas celui de l'enfant lui-même, mais bien, comme le souligne par exemple Élodie Kerrien (2012), celui de la tâche supposée que l'adulte pense devoir s'imposer. Le souci est, comme l'ont bien démontré les travaux de Gérard Mendel (1971), que la prématurité du nouveauné implique pour lui la mort si aucun adulte ne parvient à prendre soin de lui. C'est ce qui rend le désinvestissement potentiellement filicide, non pas par intention délibérée mais par réalité de la précarité du nourrisson. 
$C^{\prime}$ est pourquoi, sur le plan social, le désinvestissement doit être rendu impossible : les femmes n'ont pas le choix, et tout un ensemble de réalités sociales se construisent pour réprimer ce risque. Mais à quel prix ? La subtilité de l'écriture de Doris Lessing ${ }^{2}$ nous permet d'en ressentir la palette complète des nuances, des ambivalences et des non-dits.

FAIRE UNE PLACE À SES ÉPROUVÉS, ET NOTAMMENT AUX ÉPROUVÉS DE TERREUR

C'est à partir des rapports de race que l'écrivaine a expérimenté cette écriture de la complexité. Son premier ouvrage, Vaincue par la brousse, inspiré de sa vie en Rhodésie du Sud (I'actuel Zimbabwe) où elle a grandi, raconte l'histoire impossible d'une jeune femme blanche et de son domestique noir. Impossible parce que leur relation est impossible, mais aussi parce que la tension de la situation et la conflictualité interne de l'héroïne rendent l'histoire elle-même impossible, impossible à vivre, au sens où aucune issue n'est possible, et même impossible à penser tant ses éprouvés (notamment ceux d'une relation a minima affectueuse avec un être qu'elle n'a pas appris à penser comme humain) ne trouvent pas place dans sa psyché. On retrouvera cette capacité à nommer l'impensable (au sens propre du terme) tout au long de son œuvre. Dans le cadre de cet article je ne pourrai, bien entendu, en donner qu'un aperçu. Pour ce faire, je me centrerai principalement sur trois œuvres de fictions (la saga des Enfants de la violence, Le carnet d'or et Mémoires $d^{\prime} u n e$ survivante) ainsi que sur ses écrits autobiographiques.

$C^{\prime}$ est par exemple dans le second tome de son autobiographie qu'elle évoque sa rencontre avec un haut dignitaire soviétique :

" Il était entouré d'aides que je pris pour des membres de son état-major, en réalité, bien sûr, des agents du KGB [...] il parlait exclusivement en jargon communiste : "La classe ouvrière, les impérialistes fascistes..." [...] $\mathrm{J}$ 'avais froid, et mes paumes étaient moites de sueur [...] J'étais effrayée. Terrifiée. Je mourrais de peur. Cela ne m'est jamais arrivé depuis " (La marche dans l'ombre, p. 142).

Ainsi, c'est la parole désincarnée qui provoque et transmet la terreur, au point que l'auteure poursuit par : « Je pense qu'à ce moment-là je me suis trouvée au plus près - à portée de main - des horreurs meurtrières de I'Union soviétique " (ibid.). "À portée de main " est à prendre au sens propre : c'est parce qu'elle en ressent la teneur d'évacuation du sujet dans la façon dans laquelle ces paroles lui sont adressées qu'elle peut ressentir la façon dont le sujet va être évacué du Goulag. Pourtant, un tel ressenti ne peut être considéré comme une analyse politique :

"Je ne discutai avec personne de cet épisode. Il était trop "subjectif", comme disaient les camarades de tout événement impossible à expliquer

2. Relayée par les traductions, notamment celles de Marianne Véron. 
dans l'immédiat. Malheureusement, certaines des rencontres les plus importantes de notre vie, qui nous transforment, paraissent si insignifiantes qu'on ne prend même pas la peine de les mentionner. Je ne retournai jamais aux réceptions de l'ambassade soviétique " (ibid., p. 142-143).

Peut-être peut-on considérer que c'est ainsi que la violence se transmet, à force de ne pas mentionner chaque fois que le sujet disparaît. Et l'auteure se sent aux premières loges de cette transmission, qu'elle évoque pour la première fois dans le tome $2^{3}$ des Enfants de la violence, saga ouvertement autobiographique :

"Martha était l'essence de la violence. Elle avait été conçue, élevée, nourrie, formée dans la violence. [...] Plus de quarante millions d'êtres humains avaient été massacrés, délibérément ou par négligence, par manque d'imagination [...] ils mourraient maintenant, pendant qu'elle se tenait sous l'arbre " (L'écho lointain de l'orage, p. 465).

Ce ressenti qu'elle ne cessera ensuite de traquer et aux sources duquel elle voudra remonter, cette chaîne, surtout, qu'elle s'efforcera de rompre (et peut-être faut-il entendre son œuvre à cette aune-là), elle I'origine dans I'histoire de ses parents :

"Ce couple, là, cet homme et cette femme, lorsqu'ils m'ont conçue, I'un se trouvait encore sous le choc d'une blessure par éclat d'obus, et I'autre en dépression nerveuse d'avoir soigné tant de blessés. Elle, Martha, était autant I'enfant de la Première Guerre mondiale que d'Alfred et May Quest"(ibid.).

Martha, c'est Doris. Elle évoquera ainsi qu'il « existe sans nul doute dans mon esprit un schéma selon lequel l'ordre est englouti par le chaos et l'excès ultimes. Cela vient de la Première Guerre mondiale, qui a provoqué I'anéantissement de mes parents " (La marche dans I'ombre, p. 316). Ce qui va nous intéresser alors est la façon dont elle nous transmet cette transmission. Car il ne s'agit pas de violence physique, affichée, visible, identifiée mais de celle, beaucoup plus sourde et non moins pernicieuse, des réseaux d'obligations et d'injonctions qui emprisonnent et terrorisent, dont Doris Lessing nous montre (ou plus exactement nous fait ressentir) la teneur (je dirais même : sa " texture "). II ne s'agit jamais de juger des personnes mais de décrire des situations et des engrenages dans lesquels tout un chacun est susceptible de se retrouver. C'est un appel à la vigilance, pas une condamnation. On comprend mieux ses démêlés avec certains " camarades "...

Tout au long des Enfants de la violence, elle décrit ce dans quoi son héroïne est prise, notamment dans son souci de construire son chemin propre, entre obligations sociales, pressions militantes et enfermement

3. Pour ce qui concerne l'édition française, en trois tomes (qui eux-mêmes peuvent différer selon les éditions successives) alors que l'édition originale anglaise en comprend cinq. La pagination fait référence à la première édition chez Albin Michel (voir bibliographie). 
sexué. J'ai été particulièrement interpellée par la finesse de la description des processus de décisions, à l'opposé des mises en balance rationnelles que l'on imagine d'ordinaire ${ }^{4}$, tel le passage où, dans cette Angleterre en déroute qu'elle imagine pour clore la saga, un responsable d'une petite communauté témoigne de la façon dont il peut, en même temps, assumer l'idée qu'il ne reste " au mieux que quelques années de survie à l'Angleterre " tout en envisageant "le moyen d'acquérir une maisonnette située juste en face de chez nous " et les possibilités de la meubler... (La cité promise, p. 586). La lutte contre la violence est peut-être là, dans le désir de maintenir, au moins dans I'imaginaire, quelque chose d'un monde en voie de s'écrouler.

\section{LE RISQUE FILICIDE}

Cette incursion vers le fantastique se poursuit dans Mémoires $d^{\prime}$ 'une survivante, où elle reprend la thématique d'une communauté en lutte pour sa survie en la liant à la question de l'accompagnement des plus jeunes. Dans un monde en pleine désorganisation, l'héroïne découvre qu'un des murs de son appartement peut s'ouvrir, lui donnant ainsi accès à un autre espace, qui est à la fois une suite mouvante de pièces qui se composent et se décomposent et le lieu de vie d'une famille. Dans le même temps, elle se voit confier une jeune fille d'une douzaine d'années, Emily. La grande force de Doris Lessing est de ne pas s'attarder sur des supposées explications, tentatives de compréhension, etc. pour se concentrer non pas sur le pourquoi mais sur le comment : un jour, vous êtes en charge de quelqu'un, et vous devez faire avec cela. Que se passe-t-il alors pour vous ? La réponse de Doris Lessing est que, comme le monde extérieur ne tient plus, il ne vous reste plus qu'à explorer les profondeurs, les vôtres et celles de l'être dont vous avez la charge. $C^{\prime}$ est ainsi que I'on peut comprendre les pièces mouvantes à restaurer comme l'exploration de la capacité restauratrice de I'héroïne, tandis que les scènes familiales sont explicitement situées comme représentant I'enfance d'Emily. Le mur s'ouvre sur la résultante des espaces psychiques générés par les deux personnages en présence.

$C^{\prime}$ est alors, derrière ce mur, qu'a lieu une scène que je qualifie de " fondatrice " et à propos de laquelle elle dira dans le premier tome de son autobiographie : " J'utilisai la nursery de Téhéran ${ }^{5}$ et les personnages de mes parents, exagérés et grossis " (Dans ma peau, p. 42). Emily, c'est donc Doris, mais c'est aussi sans doute sa mère, puisque l'auteure a choisi de donner à son personnage le prénom même de sa génitrice, petite fille

4. Dans la nouvelle "George le Léopard ", la dernières du tome 1 des Nouvelles africaines, elle évoque ainsi : "En lui-même une décision se faisait voie, et il l'abritait tendrement, ne sachant pas encore ce qu'elle serait » (p. 311).

5. Où elle a passé les premières années de sa vie. 
elle-même mal accueillie. À quoi est-elle confrontée alors, cette Emily personnage qui porte en elle à la fois la mère et la fille ? La scène que je qualifie de "pivot " (sans doute parce qu'elle me touche particulièrement) représente un moment en apparence banal de "jeu », préconisé par la mère, entre le père et l'enfant, mais elle tourne vite au cauchemar ${ }^{6}$ :

" L'homme à I'allure militaire était assis et tenait entre ses genoux écartés la petite fille hurlante [...] II "chatouillait" I'enfant [...] II fallait jouer avec l'aînée, la fatiguer, lui donner son dû d'attention avant de la coucher, et le père rendait ce service à la mère [...] La pièce semblait pleine d'angoisse brûlante, de la peur de rester attachée là, du besoin d'être retenue et torturée, puisque c'était ainsi qu'elle satisfaisait son ravisseur. Elle hurlait $[\ldots]$ impuissante, tandis que cet homme l'explorait et la dévoilait ${ }^{7}$ " (Mémoires d'une survivante, p. 92).

\section{Que se passe-t-il, pendant ce temps-là, pour les adultes ?}

" La mère [occupée avec son bébé] restait indifférente [...] Car il s'agissait d'un "jeu", et comme les cris de protestation venaient de sa propre enfance, ils étaient normaux, sains, permis [...] Le père poursuivait sa tâche et, de temps à autre, regardait sa femme avec une expression remarquablement complexe - de culpabilité, mais il ne s'en rendait pas compte ; de supplication parce que tout cela lui semblait déplacé et qu'il aurait voulu cesser ; d'étonnement que ce fût permis [...] ; et puis, mêlée à tout cela, une expression qui ne disparaissait jamais bien longtemps de son visage, de pure incrédulité devant l'impossibilité des choses"(ibid.).

Cette "impossibilité des choses ", c'est bien entendu celle des horreurs de la guerre. Si un tel massacre a pu être possible, alors une petite fille hurlante est une chose dont on doit pouvoir s'accommoder...

«Mais tout cela - et le combat pour la survie - se poursuivait parallèlement aux activités organisées avec compétence et intelligence par ma mère, la fille de John William, qui lui avait enseigné ce qu'un bon parent devait procurer à un enfant "(Dans ma peau, p. 45).

Car là est le piège central, dans l'obligation que la mère se donne sans parvenir à l'assumer. S'ensuit une longue plainte, particulièrement destructrice pour l'enfant :

«Malheureusement ce genre de conversation se déroule devant les enfants, comme s'ils n'étaient pas présents et ne l'entendaient pas dire au monde qu'ils sont un poids, une déception, et lui prennent toutes ses forces [...] combien tous ses talents s'étiolaient, inexploités, combien la petite fille en particulier (si difficile, si vilaine !) transformait sa vie en calvaire. Une haine glacée $\mathrm{m}^{\prime}$ embrasait, j'aurais pu la tuer sur place. Puis j'éprouvais une lassitude, une amertume [...] je savais que cela ne plaisait pas à mon père : je percevais ce qu'il sentait. II souffrait à cause de sa femme, cette solide masse insensible qui ne semblait pas savoir ce qu'elle faisait " (ibid., p. 42).

6. L'auteure s'attardera longuement sur ce terme dans son autobiographie.

7. La dimension incestuelle est soulignée un peu plus loin. 
La mère, pourtant, assume, dans un processus que sa fille résumera dans une interview :

" He just drifted off, he couldn't cope. My mother was the organizer, and kept everything together [Mon père a juste dérivé, il ne pouvait pas faire face. Ma mère était l'organisatrice, et gardait tout ensemble ${ }^{8}$ ] » (interview par Thomas Frick).

Elle évoquera par ailleurs la « maison extraordinaire que [sa] mère avait créée ${ }^{9}[\ldots]$ à partir du contenu de quelques malles bourrées d'achats de chez Liberty et Harrods " (Alfred et Emily, p. 256). Ce " garder tout ensemble ", qui me semble central, se fait avant tout en reproduisant le déjà connu.

\section{LE REFOULEMENT DU DOUTE ET DES AMBIVALENCES}

$C^{\prime}$ est à cet inlassable travail de rassemblement que Doris Lessing s'attellera, via l'écriture, en tâchant d'y consacrer le temps et l'énergie nécessaires pour le faire sans évacuer le sujet. Car c'est bien cela la souffrance : un monde organisé pour lutter contre le chaos et la désespérance et pouvoir malgré tout y faire grandir les enfants, à partir d'un déjà connu impossible à reproduire et ne pouvant donc prendre en compte I'ensemble des vécus des protagonistes. Un monde où la stabilité doit I'emporter coûte que coûte, mais à quel prix ? "Une pièce qui oppressait et décourageait à force de proclamer que chaque chose ici avait sa place et son heure, et que rien ne pouvait changer ou échapper à cet ordre immuable " (Mémoires d'une survivante, p. 75).

Un élément me semble central dans la plainte et la culpabilité de la mère : ce sont les aspirations littéraires auxquelles elle doit renoncer et qu'elle fait rejaillir sur l'enfant. Car c'est dans ses exigences de sujet, désireuse elle aussi de liens et de compréhension du monde, que la petite fille est le plus insupportable :

"Je sais qu'Emily aurait besoin de plus que je ne peux lui donner, mais c'est une enfant si difficile, exigeante, elle m'a toujours vidée, il faut lui faire la lecture et jouer avec elle sans relâche " (ibid., p. 74).

Comment transmettre ce à quoi on est soi-même en train de renoncer ?

"Elle était prise au piège mais ne comprenait pas pourquoi, car son mariage et ses enfants constituaient le but, l'aspiration intime de sa vie - ce que la société avait choisi pour elle. Rien dans son éducation ni dans son expérience ne l'avait préparée pour ce qu'elle éprouvait réellement et elle était emmurée dans sa détresse et sa déception " (ibid., p. 75).

Enfermée dans une économie psychique de survie, la mère va alors transmettre "I'impossibilité des choses ". Ainsi, lorsque la fillette insiste

8. Traduction personnelle.

9. En pleine brousse africaine. 
pour un câlin, elle cède, dans un subtil mélange de narcissisme et d'injonction surmoïque :

"Le devoir la faisait rester [...] entre le corps chaud et plein d'envie ardente de l'enfant qui aspirait à se faire apaiser d'une caresse tendre [...] entre elle et le corps calme et régulier de la mère, entièrement voué à l'efficacité et au devoir, s'étendait un vide, une absence : il n'existait aucun contact, aucun confort mutuel " (ibid., p. 96-97).

Insistons encore une fois : il ne $s^{\prime}$ agit pas de culpabiliser mais de décrire. L'auteure elle-même (directement ou à travers ses personnages) $s^{\prime}$ inclut dans ces femmes qui ne parviennent pas à assumer la charge de leurs enfants comme elles le souhaiteraient : " Je la regardais en pensant : c'est mon enfant, ma chair et mon sang. Mais je ne le ressentais pas [...] c'est parce que j'éprouvais une hostilité à l'égard de Michael - et ça paralysait tout "(Le carnet d'or, p. 354-355).

\section{CONTRE LE DÉNI DU DÉSIR : FAIRE VIVRE LA MULTIPLICITÉ EN SOI}

Une citation du même Carnet d'or, où l'héroïne, Anna Wulf, expose sa conception de la vie à Tommy, le fils adolescent de son amie Molly, éclaire singulièrement I'ensemble :

«Ce n'est rien de terrible - je veux dire, ce peut être terrible mais ce n'est pas destructif, ce n'est pas venimeux, de se passer de ce qu'on veut. II n'y a rien de mal à dire : mon travail ne correspond pas réellement à ce que je veux [...]. Ou bien : j'ai besoin d'amour mais je m'en passe. Ce qui est terrible, c'est prendre la médiocrité pour la grandeur. C'est de prétendre qu'on n'a pas besoin d'amour alors que c'est faux; ou de prétendre que I'on aime son travail alors qu'on se sait capable de mieux. Je me trouverais bien coupable de prétendre, par honte ou autre chose, que j'ai aimé le père de Janet alors que ce n'est pas vrai " (p. 398).

La violence, pour Doris Lessing, ce n'est pas de ne pas vivre la vie qu'on voudrait. C'est de ne pas pouvoir le conscientiser, l'exprimer et I'assumer. Car rien n'est pire que la distorsion du désir, c'est-à-dire ce qui est donné sans être donné et prétend répondre à la demande d'autrui.

Mais comment faire face lorsque le monde s'écroule ? Comment échapper au chaos, parvenir à trier et organiser, tout en le faisant à partir de la réalité du sujet et pas contre elle ? Travail titanesque auquel les femmes sont vouées depuis la nuit des temps, qu'elles réussissent plus ou moins bien selon les circonstances et qui est rendu d'autant plus difficile par la logique de la violence, c'est-à-dire celle où la résolution du conflit se fait par l'élimination d'une des parties en présence.

Ce que nous montre Doris Lessing, c'est donc à la fois la difficulté et la nécessité d'écouter et de faire vivre en soi la conflictualité et la multiplicité, la nôtre et celle de nos enfants. "Lorsqu'une femme considère un enfant, elle retrouve en même temps tout ce qu'il a été " (Le carnet d'or, p. 400). "Écouter " signifie ici « entendre I'inaudible », dont le risque 
filicide est sans doute l'archétype. Si I'on considère la notion d'éradication du doute (Hatchuel, 2013a) que j'ai évoquée en introduction et qui me semble caractériser le rapport au savoir des sociétés hypermodernes, Doris Lessing nous montre ici que soutenir le désir c'est avant tout soutenir sa complexité, y compris dans ce qu'elle comporte de doute. Cette prise en compte de la complexité passe par un processus dont on retrouvera une description extrêmement fine tout au long de l'œuvre et qui peut se résumer en trois verbes : reconnaître, séparer et relier.

La première étape réside donc dans I'ancrage sensoriel. II peut s'agir de retrouver des sensations perdues ("Passé la matinée à essayer de me rappeler la sensation d'être assise sous les arbres, dans le v/ei ${ }^{10}$ près de Mashopi. Raté ", écrit ainsi Anna, I'héroïne du Carnet d'or, p. 110) ou de trouver les formes pour exprimer l'inexprimable, tel ce tigre qui m'apparaît comme une mise en forme fantasmatique de la terreur et autour duquel tourne l'ensemble de rêveries, diurnes et nocturnes, qui occupe une très grande partie de la fin du Carnet d'or, envahissant littéralement l'espace, physique et psychique.

Vient ensuite le travail de séparation. À cet égard, le dispositif autour duquel est construit ce même Carnet d'or constitue l'image la plus parlante : il s'agit en effet d'un roman intitulé Femmes libres, centré autour de I'histoire d'Anna Wulf, écrivaine, de son amie Molly et de leurs proches, qui peut être lu indépendamment, mais qui est entrecoupé des quatre carnets qu'Anna tient à jour et qui, chacun, recouvre une partie de sa vie et de ses activités. Le carnet d'or est celui qui vise à réunir les quatre autres (" moi tout entière dans un seul carnet ", p. 868). "Pourquoi ces quatre carnets ? Qu'arriverait-il si tu avais un seul gros cahier? ", demande ainsi Tommy à Anna. "Je te I'ai dit, ce serait le chaos " (p. 406).

Mais la notion se retrouve également dans l'utilisation de l'espace, et notamment de l'espace domestique, véritable mise en scène du psychisme. Au fil des différents romans courent des images de maisons collectives ou voisines truffées de sous-sols ou de dépendances, de chambres au sein d'appartements, d'espaces " annexes " comme dans La cité promise (tome 3 des Enfants de la violence) où Martha Quest prend la place d'une maîtresse de maison de retour d'une hospitalisation psychiatrique, notamment pour veiller sur son fils, tandis que celle-ci s'installe dans un appartement au sous-sol. Comment ne pas penser aux multiples personnalités d'une même femme, tantôt en capacité d'assumer les tâches qui lui incombent et tantôt non ? C'est dans ce même esprit que j'entends les similitudes (de situations, de prénoms, d'interrogations) qui se répondent au fil de l'œuvre. D'un roman à l'autre,

10. Terme afrikaans désignant un lac intermittent. Marianne Véron choisit de ne pas le traduire. 
ce sont toujours les mêmes enjeux et en même temps chaque histoire se suffit à elle-même.

On retrouve cela avant tout dans Le carnet d'or, que I'on peut considérer comme centré sur la permanente réécriture des mouvements émotionnels et psychiques (les mouvements psychiques étant constitués de la résultante des émotions et des sensations) de son héroïne, qui est ainsi conduite à retrouver, assumer puis stabiliser, au moins momentanément, ces mouvements. On peut en effet considérer que Femmes libres, le "roman dans le roman " du Carnet d'or, tourne autour des différents personnages, de la vie sociale, de ce qui peut être affiché, tandis que les différents carnets occupent chacun une fonction précise : le carnet bleu se centre sur les préoccupations émotionnelles du moment d'Anna Wulf (notamment la relation avec I'homme avec qui elle a une liaison au cours du roman et avec lequel elle relate la rencontre interpsychique avec une précision d'entomologiste) ; le carnet jaune, qui recueille plusieurs propositions de romans ou nouvelles, partiellement rédigés ou sous forme de synopsis, dans lesquels on retrouve plusieurs situations et personnages de la vie de I'héroïne mais aussi de celle de Doris Lessing, montre ce qu'elle pourrait essayer d'en faire ; le carnet noir, qui comprend notamment le récit des démêlés avec les éditeurs et adaptateurs, peut être vu comme une façon de rendre compte des incompréhensions que suscitent ces tentatives de transmission; Femmes libres montre ce qu'elle en fait finalement. La boucle est bouclée. Ainsi, la même scène constituera d'abord un projet de roman dans le carnet jaune (p. 765) avant d'être "relatée " dans le carnet bleu (p. 805), mais elle n'apparaît plus dans Femmes libres. Anna Wulf souligne d'ailleurs $q u^{\prime}$ " il est évident que la manière dont je transforme tout en fiction n'est qu'un moyen de me dissimuler à moi-même quelque chose " ( $p$. 348). Le magistral tour de passe-passe est de montrer que c'est ce retour au plus archaïque du psychisme qui rend possible l'écriture " sociale " mais que celle-ci gommera cela.

Si I'on se penche sur la chronologie des écrits, on repère que Doris Lessing écrit les deux derniers tomes des Enfants de la violence (qui datent respectivement de 1965 et 1969 , alors que le premier tome est sorti en 1958) après Le carnet d'or (1962). On peut donc considérer que c'est seulement après avoir mis au travail cette circulation de sa réalité émotionnelle qu'elle peut terminer le récit de son expérience africaine et de son arrivée à Londres, avant de s'autoriser une incursion du côté du fantastique, genre qu'elle reprendra à plusieurs reprises, avec à chaque fois une liberté étonnante dans la facilité avec laquelle elle franchit les frontières entre le réel et I'imaginaire et qui constituera le dispositif central de Mémoires d'une survivante (1974). 
La dernière étape sera celle du rassemblement où les différentes parties (d'un même sujet, de l'image de l'autre, d'une maison) pourront se relier et cohabiter. À plusieurs reprises, notamment dans Le carnet $d^{\prime}$ or, l'auteure évoque ces personnages qui ne savent plus à qui ils ont affaire, I'autre et eux-mêmes traversant en simultané des états différents et multiples. On peut imaginer alors que la saisie de cette complexité se fait à partir de cet étonnant «jeu " auquel se livre Anna depuis son enfance. Sa description nous permet de comprendre le travail psychique nécessaire à cette cohabitation des différentes dimensions au sein du psychisme, où chaque élément existe à la fois pour lui-même et comme partie d'une continuité, permettant une conscience de soi dans le monde et ses multiples niveaux ${ }^{11}$ :

« D'abord je créais la pièce dans laquelle j'étais assise, objet par objet, en "nommant" chaque chose, le lit, la chaise, les rideaux [...] puis je quittais la pièce pour créer la maison et, lentement, créais la rue [...] puis lentement, très lentement, je créais le monde, continent par continent, océan par océan (mais le but du jeu consistait à créer cette immensité tout en gardant à l'esprit ma chambre, la maison et la rue dans leur petitesse [...] Et, comme j'avais atteint ce point [...] j'essayais d'imaginer en même temps une goutte d'eau grouillante de vie ou une feuille verte " (p. 785-786).

L'Emily de Mémoires d'une survivante est accueillie par une adulte qui n'est pas sa mère et peut ainsi regarder son passé avec sérénité puisqu'elle n'en est pas la cause, tout en y portant attention du fait du souci qu'elle a de l'adolescente. Peut-être serait-ce alors cela, le care, le " prendre soin » que les théoriciennes du genre (Tronto, 2009 ; Molinier, 2013) recommandent de mettre au centre du lien social : prendre ensemble la totalité des sensations et émotions qui se nouent entre soi et I'autre à partir notamment des passés respectifs, et parvenir à leur faire prendre forme. Le care, ce serait permettre à l'autre de ne jamais céder non seulement sur son désir mais sur son ressenti et sa complexité, même s'ils ne peuvent se réaliser : comme le dit Anna à Tommy, ne pas réaliser son désir peut être terrible, le tordre est destructeur. La violence, ce serait alors l'échec du care.

En reprenant la notion de "fiction de soi " sur laquelle j'avais centré mon habilitation à diriger les recherches (Hatchuel, 2013b) sans faire explicitement référence à Doris Lessing mais après I'avoir beaucoup lue ${ }^{12}$, je dirai aujourd'hui que prendre soin de l'autre, ce serait peut-être I'aider à construire sa fiction de soi. Cela signifierait non seulement que le sujet doit à la fois être interprété et être institué comme pouvant

11. On pourra également se reporter à la très éclairante réflexion de Janine Puget sur les "mondes superposés" (voir par exemple Puget, 2006 et 2015).

12. Je tiens ici à remercier Sylvie Barbier, la bibliothécaire qui me I'a fait découvrir. 
s'écarter de cette interprétation, en accord avec la théorisation de Piera Aulagnier (1975), mais aussi que ce qui doit être également institué, c'est I'idée même que l'histoire est mouvante et peut et doit être remaniée. Un peu à la façon dont René Kaës (2005) définit le groupe interne, c'est-à-dire la conception que chacun de nous a du groupe à partir de ses différents groupes internes, je considère la fiction de soi comme l'idée même des histoires que nous nous racontons sur nous à partir de celles qui nous ont été racontées et remaniées. II s'agit donc de se penser et penser l'autre avec les cadres que l'extérieur a prédéfinis mais sans s'y limiter.

\section{CONCLUSION}

Les femmes rangent le monde comme elles peuvent, réparent, font tenir... Parfois elles y arrivent, parfois non. Doris Lessing nous propose de sortir du double piège du moralisme et de l'idéalisme. Dénonçant I'illusion d'un monde qui serait sans souffrance, sans conflit, sans frustration (déni qui constitue le meilleur moyen de nous empêcher d'y faire face), elle propose de renoncer aux professions de foi, de dire non pas comment nous voudrions que le monde soit mais comment il est avant éventuellement de l'imaginer autrement.

Contre la logique de la culpabilité, et en cohérence avec le refus de la résolution du conflit par l'éradication, elle dénonce des systèmes, jamais des personnes. Elle souligne que personne ne peut penser la vie $d^{\prime}$ autrui mais que penser sa propre vie est une tâche quotidienne, un inlassable travail psychique qui n'est possible qu'à condition de ne pas s'obliger, dans un sursaut volontariste ou démesurément inquiet, à prendre en charge ce que I'on est en fait incapable d'assumer. Dans un monde où l'intériorité se construit de moins en moins, il s'agit avant tout de $\mathrm{s}^{\prime}$ autoriser à revenir à soi. Doris Lessing en affirme I'absolue nécessité.

\section{FUVRES DE DORIS LESSING}

La bibliographie est classée par ordre chronologique de parution des éditions originales. Pour chaque ouvrage, outre cette date de parution originale, on trouvera la traduction et la première édition en France. Sauf mention contraire, c'est celle à laquelle il est fait référence pour les paginations des citations. Les ouvrages cités dans le présent article sont en gras.

La bibliographie complète est disponible sur : http://www.dorislessing.org

On peut trouver le détail de certaines éditions sur http://soumbala.com (site sur la littérature africaine).

Vaincue par la brousse, 1950, trad. Doussia Ergaz, Plon, 1953.

Nouvelles africaines, à partir de 1951, trad. Marianne Véron, en deux tomes chez Albin Michel, en trois au Livre de Poche. L'édition consultée est celle de 2012 au Livre de Poche. 
Les enfants de la violence

- $\quad$ Première parution en France du tome 1 (Martha Quest), trad. Doussia Ergaz et Florence Cravoisier, Plon, 1957.

- Repris chez Albin Michel (trad. Marianne Véron) :

T1 Les enfants de la violence, 1978 ; regroupe Marta Quest (1952) et A propper mariage (1954).

T2 L'écho lointain de l'orage, 1979 ; regroupe $A$ Ripple from the Storm (1958) et Landlocked (1965).

T3 La cité promise, 1981, The Four-Gated City (1969).

Retreat to innocence,1956, non traduit.

Plusieurs essais, nouvelles et poèmes.

Going home,1957, non traduit.

L'habitude d'aimer, 1957, trad. Marianne Véron, Albin Michel, 1992.

Pièces et essais dont London Diary.

Un documentaire.

Le carnet d'or, 1962, trad. Marianne Véron, Albin Michel, 1976. L'édition consultée est celle du Livre de Poche, 2011.

Play with a Tiger, 1962, pièce non traduite.

Traductions, essais, nouvelles, plusieurs pièces....

Un homme et deux femmes, 1963, trad. Jacqueline Marc-Chadourne, Plon, 1967. Descente aux enfers, 1971, trad. Pierre Alien, Albin Michel, 1988.

L'été avant la nuit,1973, trad. Marianne Véron, Albin Michel, 1981.

Mémoires d'une survivante, 1974, trad. Marianne Véron, Albin Michel, 1982.

L'édition consultée est celle du Livre de Poche, 1996, rééditée en 2016.

Canopus in Argo, 1979, 1980, 1981, 1982, 1983, deux premiers tomes traduits au Seuil, 1981 et 1983.

Les carnets de Jane Sommers, 1983 et 1984, trad. Marianne Fabre (T1) et Nathalie Zimmermann (T2), Albin Michel.

Filles impertinentes, 1984, trad. Philippe Giraudon, Flammarion, 2014.

La terroriste, 1985, trad. Marianne Véron, Albin Michel, 1986.

Le cinquième enfant, 1988, trad. Marianne Véron, Albin Michel, 1990.

Autobiographie : T1 Dans ma peau,1994, et T2 La marche dans I'ombre, 1997, trad. Anne Rabinovitch, Albin Michel, 1995 et 1998.

L'amour encore, 1996, trad. Anne Rabinovitch, Albin Michel, 1996.

Mara et Dann,1999, Le cycle de I'eau T1, trad. Isabelle Delord-Philippe, Flammarion, 2001.

Le monde de Ben, 2000, trad. Marianne Véron, Flammarion, 2000.

Le rêve le plus doux, 2001, trad. Isabelle Delord-Philippe, Flammarion, 2003.

Les grands-mères, 2003, trad. Isabelle Delord-Philippe, Flammarion 2005.

Victoria et les Staveney, 2003, trad. Philippe Giraudon, Flammarion, 2010.

Un enfant de I'amour, 2003, trad. Isabelle Delord-Philippe, Flammarion, 2007.

Le temps mord, 2004, trad. Philippe Giraudon, Flammarion, 2011.

L'histoire du général Dann, 2005 Le cycle de l'eau T2, trad. Philippe Giraudon, Flammarion 2013.

Comment ne pas gagner le Prix Nobel ? Discours de réception du prix Nobel 2007, trad. Isabelle Delord-Philippe, [en ligne] http://www.lemonde.fr/idees/article/ 2007/12/08/comment-ne-pas-gagner-le-prix-nobel-par-doris-lessing_987394_ 3232.html 
Alfred et Emily, 2008, trad. Philippe Giraudon, Flammarion, 2008. L'édition consultée est celle du Livre de Poche, 2009.

\section{Autour de Doris lessing : émissions et interviews}

Apostrophe du 25 décembre 1981, entièrement consacré à Doris Lessing http://www.ina.fr/video/CPB81053226/doris-lessing-video.html

Frick, T. 1988. "The Paris review. Doris Lessing ", The Art of Fiction, ${ }^{\circ} 102$, http://www.theparisreview.org/interviews/2537/the-art-of-fictionno-102-doris-lessing

\section{BIBLIOGRAPHIE}

AULAGNIER, P. 1975. La violence de l'interprétation : du pictogramme à l'énoncé, Paris, Puf.

AULAGNIER, P. 1982. "Condamné à investir ", Nouvelle revue de psychanalyse, 25, 309-330. Repris dans Un interprète en quête de sens, Paris, Ramsay, 1986, 325-358.

DIET, E. 2003. « L'homme procédural. De la perversion sociale à la désubjectivation aliénante ", Connexion, 79, 11-27.

GAILLARD, G. 2001. "La généalogie institutionnelle et les écueils du travail d'historisation : entre filicide et parricide ", Connexions, 76, 125-141.

HATCHUEL, F. 2004. "Le groupe de paroles d'apprenants et d'apprenantes : un espace de co-formation ? ", Connexions, 82, 143-157.

HATCHUEL, F. 2007. "Doris Lessing, la force obscure des femmes ", Esprit, 238-241

HATCHUEL, F. 2009. Du rapport au savoir à la fiction de soi : penser, vivre et faire grandir dans un monde incertain. Anthropologie clinique de la transmission, Note de synthèse en vue de l'habilitation à diriger les recherches, université Paris Ouest Nanterre La Défense.

HATCHUEL, F. 2012. "Soutenir le travail : une posture psychique face au chaos", Connexions, 97, 119-135.

HATCHUEL, F. 2013a. "Rapport au savoir, besoin de croire et illusions de certitudes. De quelques caractéristiques hypermodernes ", Nouvelle revue de psychosociologie, 16, 113-125.

HATCHUEL, F. 2013b. "Modalités d'écriture en situation de formation clinique : élaborer le rapport à I'interlocuteur interne ", Cliopsy, 10, 53-66.

KAËS, R.1984. "Étayage et structuration du psychisme ", Connexions, 44, 11-46.

KAËS, R. 2005. "Groupes internes et groupalité psychique : genèse et enjeux d'un concept ", Revue de psychothérapie psychanalytique de groupe, 45, 9-30.

KERRIEN, E. 2012. L'enfant rom dans l'imaginaire enseignant. D'une approche psychosociale à une approche clinique de l'Autre, Mémoire de master recherche de sciences de l'éducation, sous la direction de Françoise Hatchuel, université Paris Ouest Nanterre La Défense.

MendEL, G. 1971. Pour décoloniser l'enfant : sociopsychanalyse de l'autorité, Paris, Payot.

MOLINIER, P. 2013. Le travail du care, Paris, La Dispute.

PUGET, J. 2006. "Penser seul ou penser avec un autre ", Revue de psychothérapie psychanalytique de groupe, 46, 31-40. 
PUGET, J. 2015. "Le faire ensemble et avec soi-même. Deux contextes superposés ", Revue de psychothérapie psychanalytique de groupe, 64, 67-77.

TRONTO, J. 2009. Un monde vulnérable. Pour une politique du care, Paris, La Découverte.

FRANÇOISE HATCHUEL, DU RISQUE FILICIDE AU CARE : LA TRANSMISSION DE LA VIOLENCE ET SON INTERRUPTION DANS L'CEUVRE DE DORIS LESSING

RÉSUMÉ

À travers quelques-uns de ses ouvrages (principalement les trois tomes du cycle des Enfants de la violence, les deux romans intitulés Le carnet d'or et Mémoires $d^{\prime}$ 'une survivante et trois écrits à caractère autobiographique), I'article se propose d'étudier la façon dont l'écriture de Doris Lessing, prix Nobel de littérature 2007, met en forme et en scène la transmission de la violence. La question centrale se noue autour de ce que l'auteure de l'article appelle "le risque filicide ", c'est-àdire le risque du désinvestissement (au sens de Piera Aulagnier), potentiellement mortel pour les enfants, et donc impossible à assumer. Ce sont les effets des injonctions contradictoires qui en résultent que l'article s'efforce de repérer, montrant comment le care, le " prendre soin " des enfants, pourrait consister à assumer la complexité des mouvements émotionnels qui nous relient à eux.

MOTS-CLÉS

Doris Lessing, transmission de la violence, risque filicide, care.

FRANÇOISE HATCHUEL, FROM RISK OF FILICIDE TO CARE:

VIOLENCE TRANSMISSION AND INTERRUPTION IN DORIS LESSING WORK

\section{ABSTRACT}

This article, based on few of her workpieces (three volumes of the "Children of violence" series, two novels entitled "The golden notebook" and "Memoirs of a survivor" and three autobiographical pieces), explores the way Doris Lessing's writing shapes and stages violence transmission. The core question is what the article's author calls "risk of filicide", which is the risk of disinvestment (as Piera Aulagnier defined it), potentially mortal for children and therefore impossible to assume. This can generate contradictory injunctions. The purpose of the article is to identify them and to show its effects on the relationship onto the child, demonstrating how taking care of children could be about taking into account the complexity of the emotional movements which linking us to them.

\section{KEYWORDS}

Doris Lessing, transmission of violence, risk of filicide, care. 